102

A NOVEL APPROACH TO ADDRESSING FIBROMYALGIA SYMPTOMATOLOGY IN SLE

Jennifer L Rogers*, Amanda M Eudy, Lisa G Criscione-Schreiber, David S Pisetsky, Kai Sun, Jayanth Doss, Megan EB Clowse. Duke University

\subsection{6/lupus-2019-Ism.102}

Background The physician global assessment (PGA) is a 3point visual analog scale assessing lupus disease activity. We implemented a Type II PGA to assess what we have termed Type II SLE symptoms. Type II SLE symptoms encompass fatigue, widespread pain, depression, sleep and cognitive dysfunction, and differ from Type I SLE symptoms, such as nephritis and inflammatory arthritis, the classic manifestations of SLE inflammation.

Methods This was a cross sectional study of SLE patients (ACR 1997 or SLICC 2012 criteria). All patients completed Systemic Lupus Activity Questionnaire (SLAQ), Patient Health Questionnaire-9 (PHQ9), and 2016 ACR Fibromyalgia criteria. The authors recorded PGA, SLEDAI and Type II SLE therapy. Patients were divided into 4 groups based on lupus disease activity. Type I SLE group was defined as SLEDAI 6, clinical SLEDAI 4, or active nephritis. Type II SLE group was defined as meeting

Abstract 102 Table 1 Correlation of Type II PGA with physicianand patient-reported measures in SLE

\begin{tabular}{|c|c|c|c|c|c|}
\hline & $\begin{array}{l}\text { Low } \\
\text { Activity }\end{array}$ & $\begin{array}{l}\text { Type I } \\
\text { SLE }\end{array}$ & $\begin{array}{l}\text { Type II } \\
\text { SLE }\end{array}$ & Mixed SLE & Overall* \\
\hline & $\mathrm{n}=92$ & $\mathrm{n}=68$ & $n=18$ & $n=19$ & $\mathrm{p}$-value \\
\hline Physician & Mean (SD) & Mean (SD) & Mean (SD) & Mean (SD) & \\
\hline \multicolumn{6}{|l|}{ Assessments } \\
\hline Type I PGA & $0.5(0.7)$ & $1.1(0.6)$ & $0.4(0.4)$ & $1.2(0.6)$ & $<0.0001^{\#}$ \\
\hline Type II PGA & $0.7(0.8)$ & $0.9(0.8)$ & $2.0(0.9)$ & $1.2(0.5)$ & $<0.0001^{\#}$ \\
\hline Clinical SLEDAI & $0.4(0.9)$ & $3.9(2.9)$ & $0.3(0.8)$ & $4.7(1.8)$ & $<0.0001$ \\
\hline Full SLEDAI & $1.6(1.6)$ & $7.5(3.4)$ & $0.8(1.2)$ & $6.4(3.6)$ & $<0.0001^{\#}$ \\
\hline \multicolumn{6}{|l|}{ Patient Reported } \\
\hline \multicolumn{6}{|l|}{ Measures } \\
\hline \multicolumn{6}{|l|}{$\underline{S L A Q}$} \\
\hline $\begin{array}{l}\text { Patient disease activity } \\
\text { (range: } 0-10)^{\ddagger}\end{array}$ & $3.5(2.8)$ & $5.6(3.0)$ & $6.8(2.0)$ & $7.1(2.3)$ & $<0.0001$ \\
\hline Total SLAQ score ${ }^{\dagger}$ & $9.7(7.1)$ & $14.4(8.4)$ & $19.2(5.7)$ & $18.9(4.7)$ & $<0.0001$ \\
\hline \multicolumn{6}{|l|}{$\begin{array}{l}2016 \text { ACR Fibromyalgia } \\
\text { criteria }\end{array}$} \\
\hline Total areas of pain & $2.8(2.7)$ & $3.4(2.5)$ & $12.6(4.6)$ & $9.7(3.7)$ & $<0.0001^{\#}$ \\
\hline $\begin{array}{l}\text { Symptom severity score } \\
\text { (cognitive dysfunction, } \\
\text { fatigue, waking } \\
\text { unrefreshed) }{ }^{\dagger}\end{array}$ & $3.3(2.6)$ & $4.1(2.6)$ & $6.4(1.4)$ & $6.7(1.5)$ & $<0.0001$ \\
\hline $\begin{array}{l}\text { Fibromyalgia severity } \\
\text { score (areas of pain } \\
\text { +symptom } \\
\text { severity score) }{ }^{\dagger}\end{array}$ & $6.1(4.7)$ & $7.5(4.2)$ & $19.0(5.1)$ & $16.4(3.9)$ & $<0.0001$ \\
\hline PHQ-9 Depression & n (\%) & n (\%) & $\mathrm{n}(\%)$ & n (\%) & \\
\hline Depression & $22(30 \%)$ & $24(41 \%)$ & $15(83 \%)$ & $8(47 \%)$ & $0.0005^{\#}$ \\
\hline
\end{tabular}

*across all 4 groups; ${ }^{\#} \mathrm{p}<0.05$ Type II SLE vs Mixed SLE, ${ }^{\dagger}$ strong correlation $(>0.6)$ with Type II PGA with $(p<0.05),{ }^{\star}$ moderate correlation $(0.4-0.6)$ with Type II PGA $(p<p<0.05)$ fibromyalgia criteria. Mixed SLE group met criteria for Type I and Type II SLE. Low activity group did not meet criteria for either Type I or Type II SLE. Differences across groups were analyzed by Fishers exact test and ANOVA. A step-wise linear regression analysis examined predictors of Type II SLE treatment.

Results The authors performed a Type II PGA on 197 patients (92\% female, mean age 45.8$)$ from July to November 2018. Type II PGA strongly correlated with patient reported fibromyalgia and depression symptoms. Type II PGA did not correlate with Type I PGA or SLEDAI. Type II PGA was significantly higher in the Type II SLE and mixed SLE groups.

Type II symptoms were addressed (counseling or intervention) at $45 \%$ of visits. In a regression model predicting Type II management, factors associated with increased Type II treatment were Type II PGA (OR 2.37, CL: 1.37, 4.08) and fibromyalgia severity score (OR 1.41, CL: 1.15, 1.72). Type I PGA (OR 0.46; 95\% CI: 0.24, 0.89) was associated with decreased Type II SLE management. Compared to a cohort of 212 SLE patient visits from January to May 2018, prior to implementing the Type II PGA score, the rate of documented Type II SLE management in the Type II SLE group increased from $53 \%$ to $89 \%(p=0.03)$.

Conclusions A PGA specifically addressing Type II SLE symptoms can be implemented in practice and correlates with patient reported Type II SLE symptoms. Scoring a Type II PGA encourages the physician to address these important SLE symptoms. Further work is needed to validate the scoring.

Funding Source(s): None

\section{DEFINING THE LUPUS-ASSOCIATED PULMONARY HYPERTENSION PHENOTYPE}

Marisa Mizus*, Jessica Li, Daniel Goldman, Michelle Petri. Johns Hopkins University School of Medicine

\subsection{6/lupus-2019-Ism.103}

Background Pulmonary hypertension $(\mathrm{PH})$ is a major cause of death in systemic lupus erythematosus (SLE). Case reports and case series have identified hypocomplementemia and autoantibodies, including antiphospholipid and anti-RNP, in SLE patients with $\mathrm{PH}$. This is the first study to define the lupus phenotype associated with pulmonary hypertension $(\mathrm{PH})$ using multiple autoantibodies.

Methods 207 (8\%) SLE patients with $\mathrm{PH}$, defined as a right ventricular systolic pressure greater than $40 \mathrm{mmHg}$ on transthoracic echocardiogram or as pulmonary artery dilatation noted on computed tomography of the chest, were identified from the Hopkins Lupus Cohort (94\% female, 56.5\% African American, 39\% Caucasian, mean age 45.6 years). Agglomerative hierarchical clustering algorithm with Wards method was used to cluster the patients who had $\mathrm{PH}$, based on their history of autoantibodies. Autoantibodies used in the clustering analysis included lupus anticoagulant, anticardiolipin, anti-beta2 glycoprotein I, antidsDNA, antiSm, antiRNP, false positive (FP)RPR, antiRo, antiLa, and hypocomplementemia (C3 ever low or C4 ever low). All analyses were performed in JMP version 13.0 and $\mathrm{R}$ version 3.5 .1 using packages cluster and gplots. 

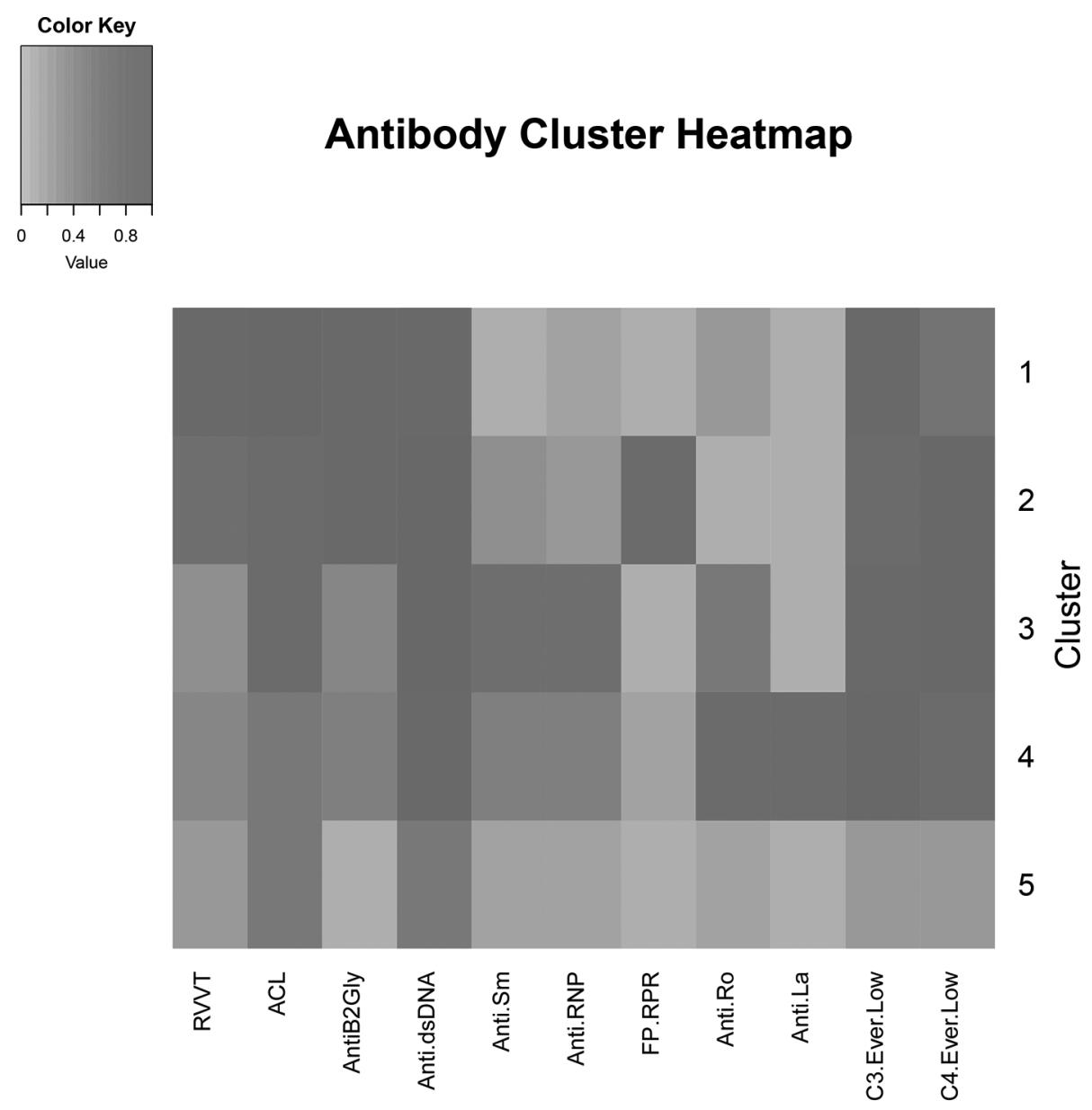

\section{Autoantibodies}

Abstract 103 Figure 1 Heatmap clustering. Heatmap shows the frequency of each autoantibody by cluster: russell viper venom test (RVVT), anticardiolipin (ACL), anti-beta2 glycoprotein I (AntiB2Gly), antidsDNA, antiSm, antiRNP, false positive (FP)RPR, antiRo, antiLa, C3 ever low, and C4 ever low. Antibody frequency is colored from grey $(0.0$, low) to red $(1.0$, high). Heatmap rows corresponding to clusters $1,2,3,4$, and 5 are indicated.

Results There were five unique clusters. Clusters 1 and 2 had high frequencies of hypocomplementemia, antiphospholipid antibodies, and anti-dsDNA. Cluster 2 additionally had a high frequency of FP-RPR. Cluster 3 had high frequencies of hypocomplementemia, anticardiolipin, anti-Sm, anti-RNP, and antidsDNA. Cluster 4 had high frequencies of hypocomplementemia, anti-Ro, anti-La, and anti-dsDNA. Cluster 5 had only moderate frequencies of anti-dsDNA and anti-cardiolipin antibody. Two clusters were defined by antiphospholipid antibodies. Two clusters were defined by anti-RNP and anti-Sm. Multiple clusters had elevated anti-dsDNA and hypocomplementemia.

Conclusions PH in SLE is defined by five unique autoantibody clusters. Antiphospholipid and anti-RNP/Sm clusters clearly separate, which likely indicates different pathophysiologic mechanisms. Four of the five clusters have antidsDNA and low complement components. Our next step is to exploreboth in the literature and within our cohortthe natural history and response to treatment of these clusters.

Funding Source(s): NIH Grant R01-AR069572

Clustering of Autoantibodies in Lupus-Associated Pulmonary Hypertension

\section{PREDICTORS OF READMISSION IN LUPUS COMPARED TO GENERAL MEDICARE PATIENTS}

${ }^{1}$ Christie Bartels*, ${ }^{2}$ Ann Chodara, ${ }^{2}$ Xing Wang, ${ }^{2}$ Fangfang Shi, ${ }^{2}$ Amy Kind. ${ }^{1}$ University of Wisconsin School of Medicine and Public Health; ${ }^{2}$ UW School of Medicine and Public Health

\subsection{6/lupus-2019-Ism. 104}

Background Medicare measures readmissions within 30 days of hospitalization as a marker of care quality across several conditions, although beyond hospital quality, neighborhood disadvantage is a known predictor of readmission. While not a reporting condition, a 2010 study noting 27\% lupus readmissions led to our interest in examining readmission in lupus versus general Medicare including the impact of neighborhood disadvantage.

Methods Using a 20\% US national Medicare sample, we identified all patients with inpatient hospitalizations 2013-14 to compare odds of 30 day readmission among patients with SLE to the general Medicare population. Inclusion required age >18 y.o., 12 mos. Medicare Part A/B (30d. post discharge), and being alive at discharge. Covariates included age, gender, race, ethnicity, prior Medicaid, comorbidity, prior hospitalization, length of stay, hospital volume, medical school 